\title{
Aspiração folicular, maturação in vitro e injeção intracitoplasmática em éguas
}

\author{
$\underline{\text { Miguel Alejandro da Silva Rua }}^{1}{ }^{*}, \underline{\text { Celia Raquel Quirino }}^{2}, \underline{\text { Aline Pacheco }}^{3}, \underline{\text { Aylton Bartholazzi }}$

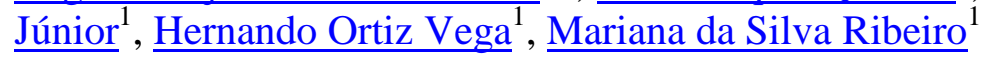 \\ ${ }^{I}$ Pós-graduando em Ciência Animal do Centro de Ciências e Tecnologias Agropecuárias, Laboratório de \\ Melhoramento Genético Animal, Universidade Estadual do Norte Fluminense Darcy Ribeiro - UENF, Campos \\ dos Goytacazes- RJ, Brasil. \\ ${ }^{2}$ Professora Associada do Laboratório de Reprodução e Melhoramento Genético Animal, Centro de Ciencias e \\ Tecnologias Agropecuárias, Universidade Estadual do Norte Fluminense Darcy Ribeiro -UENF, Campos dos \\ Goytacazes-RJ, Brasil. \\ ${ }^{3}$ Professora Universidade Federal do Oeste de Pará. Santarém,PA. Brasil. \\ *Autor para correspondência, E-mail:miguelvet-rua@hotmail.com
}

RESUMO. A utilização das biotécnicas reprodutivas tem como principal objetivo melhorar os índices reprodutivos, proporcionando o melhor aproveitamento de animais geneticamente superiores. A aspiração folicular permite a utilização de oócitos que podem ser transferidos de uma égua de boa genética para o oviduto de outra égua receptora, sendo essa receptora inseminada. A maturação de oócitos in vitro é realizada quando se realiza aspiração de folículos menores em que o oócito não esta apto a ser fertilizado. No entanto, há limitações pela falta de abatedouros para se conseguir ovários de éguas para estudos, também pelo fato da dificuldade de se realizar superovulações em éguas. Esses fatores implicam em dificuldades nos avanços dos estudos com oócitos equino. A injeção de sêmen intracitoplasmática em equinos ultrapassa a barreira da ineficiência da fertilização in vitro resultando em melhores resultados de prenhez. Há também a oportunidade de utilizar garanhões que tenham sêmen com baixa motilidade e fertilidade. As biotecnologias reprodutivas aplicadas em equinos devem ser mais estudadas para que haja aprimoramento das técnicas. Devido a grande importância da utilização das biotecnologias reprodutivas teve-se como objetivo realizar uma revisão sobre a aspiração folicular, maturação in vitro e injeção intracitoplasmática em éguas.

Palavras chave: ócito, equino, reprodução.

\section{Follicular aspiration, in vitro maturation and intracytoplasmic injection in mares}

\begin{abstract}
The implementation of reproductive biotechnologies have in orther to improve reproductive rates, favoring the production of genetically superior animals. The oocyte transfer involves aspiration of oocytes from follicles of a mare and transferring for the oviduct of another mare. The in vitro maturation of oocytes is performed when performing aspiration of follicles smaller than the oocytes in not capable of being fertilized. However, there are limitations due to the lack of slaughterhouses to achieve ovaries of mares for studies, also because of the difficulty of performing superovulations in mares. These factors imply difficulties in the advances of studies with equine oocytes. The intracytoplasmic sperm injection in the horse breaks the barrier of the inefficiency of in vitro fertilization resulting in low sperm motility and fertility. Reproductive biotechnology in horses must be studied so that there is an improvement technique. Due to the great importance of the use of reproductive biotechnologies had as purpose of reviewing on follicular aspiration, in vitro maturation and intracytoplasmic injection in mares.
\end{abstract}

Key-word: oocyte, equine, reproduction. 


\section{Introdução}

No Brasil, a equideocultura vem crescendo consideravelmente nos últimos anos, e tem sido muito importante como uma atividade lucrativa na economia do país, por gerar milhões de empregos diretos e indiretos e movimentar cerca de 7,3 bilhões de reais por ano (Escola Superior de Agricultura Luiz de Queiroz, 2006; Confederação da Agricultura e Pecuária do Brasil, 2004). Com o intuito de se obter animais com genética superior, há a necessidade da seleção de reprodutores com características desejáveis para que estas sejam herdadas pelos seus descendentes (Rodrigues, 2006).

A implantação de biotecnologias ligadas à reprodução tem como objetivo principal aprimorar os índices reprodutivos, pela utilização na reprodução de reprodutores e reprodutrizes geneticamente superiores e que apresentam características hereditárias desejáveis e eliminando da reprodução equinos que não apresentem boas características ligadas à fertilidade e que possam comprometer os índices reprodutivos do rebanho e causar prejuízo na criação (Gonçalves et al., 2001).

Com a utilização das técnicas de aspiração folicular, maturação, fertilização in vitro $\mathrm{e}$ transferência de oócitos é possível que se obtenha potros oriundos de fêmeas com padrão genético elevado (Ribeiro et al., 2008, Carnevale et al., 2010).

$\mathrm{Na}$ transferência de oócitos, realiza-se em éguas doadoras a aspiração de folículos préovulatórios e transferência do oócito para o oviduto de outra égua receptora. Os oócitos das doadoras podem ser maturados até Metáfase II in vivo ou vitro antes da fertilização. A égua receptora é inseminada e o oócito transferido é fertilizado e posteriormente ocorre o desenvolvimento do embrião dentro do aparelho reprodutivo da receptora.

Duas técnicas podem ser utilizadas para que se obtenha melhores resultados na transferência de oócitos. A coleta dos oócitos é realizada aproximadamente $12 \mathrm{~h}$ antes do tempo previsto para a ovulação e a cultura in vitro é realizada até a completa maturação prévia à transferência (Carnevale \& Ginther, 1995, Hinrichs et al., 1999, Hinrichs et al., 1998), ou coleta-se os oócitospoucos minutos antes da ovulação, com maturação completa no folículo da doadora antes de ser transferido para o oviduto da receptora
(Ray et al., 1994, Hinrichs et al., 1998, McKinnon et al., 1988). Quando se coleta os oócitos muito próximo à ovulação pode ocorrer da doadora ovular antes de ser coletada. Portanto, o método de coleta de oócitos realizado aproximadamente $12 \mathrm{~h}$ préviaà ovulação, o qual não requer cultura in vitro, é o mais utilizado.

A biotecnologia de transferência de oócitos para o oviduto da receptora é conhecido como GIFT (gamete intrafallopian transfer) o qual requer um menor número de espermatozoides, e possibilita o uso de sêmen de baixa qualidade ou de garanhões considerados subfreteis (Carnevale et al., 2000).

A aplicação da injeção de sêmen intracitoplasmática (ICSI) em equinos vem ultrapassando a barreira da ineficiência da FIV, resultando melhores resultados de prenhez utilizando oócitos que tenham sido maturados in vitro (Squires et al., 1996).

A vantagem da ICSI comparada com a FIV é que a ICSI tem a habilidade de aumentar a oportunidade de se utilizar garanhões que tenham sêmen com baixa motilidade e fertilidade in vivo.

O objetivo dessa revisão é discutir algumas biotecnologias ligadas a reprodução como a aspiração folicular, a maturação in vitro e a injeção intracitoplasmática em éguas, relatando sobre as técnicas suas dificuldades, vantagens e aplicações.

\section{Doadoras de oócito}

Éguas mais velhas e com histórico de baixa fertilidade e que tenham sido acometidas por problemas cervicais, uterinas e ovarianas e fêmeas que apresentem índices reprodutivos baixos quando submetidas à monta natural ou inseminação artificial e transferência de embriões, sem uma causa bem definida (Carnevale et al., 2005), geralmente são inseridas em programas de transferência de embriões. Normalmente, muitas dessas fêmeas apresentam resultados ruins em produzir embriões ou prenhez originadas de TE. De acordo com Squires et al. (1995) o sucesso em programas de TE é dependente da habilidade da égua em ovular, de ocorrer a fertilização no oviduto, do transporte do embrião dentro do útero eda sobrevivência do embrião até o dia da recuperação (Carnevale et al., 2000).

Uma alternativa para éguas que tem problemas ovulatórios, infecções uterinas 
frequentes, piometra ou laceração de cérvix é a utilização da transferência de oócitos. Existem algumas condições fisiológicas que interferem nas taxas de prenhez das éguas doadoras de oócitos como idade, raça, escore corporal, e variações comportamentais. Segundo Scott et al. (2001), éguas submetidas a condições de estresse ou subnutrição são doadoras com menor capacidade de produção de oócitos.

A primeira transferência de oócitos realizada com sucesso foi reportada por McKinnon et al. (1988). No entanto, apenas recentemente esta biotécnica tem sido estudada intensivamente. Carnevale \& Ginther (1995) utilizaram a transferência de oócitos com objetivo de avaliar a viabilidade de oócitos coletados de éguas doadoras mais jovens e idosas. Os mesmos autores demonstraram que quando oócitos de doadoras mais jovens eram introduzidos em ovidutos de receptoras jovens, a fertilidade era consideravelmente alta (92\%). No entanto, quando oócitos foram coletados de éguas idosas e introduzidos em ovidutos de éguas jovens, as taxas de prenhez foram consideravelmente mais baixas (31\%). Estes resultados revelaram que uma das causas da sub-fertilidade em éguas idosas está ligada a oócitos defeituosos.

Sendo assim, as éguas mais velhas seriam candidatas ao auxílio da biotecnologia de transferência de oócito devido às alterações adquiridas durante a vida reprodutiva. Carnevale et al. (2010) relataram que à medida que aumenta a idade das éguas, reduz a qualidade e a competência oocitária pela permanência destes gametas na fase diplóteno, da prófase I da meiose até o momento do recrutamento. No entanto, podem-se alcançar resultados razoáveis de prenhez utilizando a TO; no entanto, é necessário um maior número de realização da técnica para se obter um potro de uma égua idosa comparando com uma égua jovem (Carnevale et al., 2004).

\section{Utilização da gonadotrófica coriônica humana (hCG) na TO}

Para obtenção do oócitos de éguas doadoras são utilizados alguns protocolos hormonais, que tem com o objetivo acelerar a maturação folicular, assim como a maturação oocitária. Normalmente, são utilizados os hormônios hCG, hormônio liberador de gonadotrofinas $(\mathrm{GnRH})$ e o hormônio luteinizante(LH) (Squires et al., 2003).
Segundo McKinnon et al. (1997), o hormônio hCG é o hormônio mais largamente utilizado no auxiliar a maturação folicular, para diminuir o período de estro e acelerar a ovulação. Quando se detecta folículos com diâmetro de $35 \mathrm{~mm}$, a administração de hCG pode promover a ovulação em aproximadamente 40 horas (McKinnon et al., 1997).

O hormônio hCG por ser composto por moléculas glicoprotéicas de peso molecular elevado, quando é utilizado continuamente em uma mesma égua em várias estações reprodutivas, pode promover a indução de ação antigênica. Sendo assim, pode-se utilizar o GnRH e o LH como outra alternativa na indução da sincronização ovulação em éguas (Imboden et al., 2006).

Hinrichs et al. (1999) compararam as taxas de desenvolvimento de embriões provindos de oócitos coletados 24 horas após a indução com hCG e mantido em cultura por 12 horas com taxas para oócitos coletados 35 horas depois da administração do hCG e transferidos imediatamente para o oviduto da receptora. Os autores reportaram que não houve diferença na taxa de prenhez entre os dois tratamentos.

Carnevale et al. (2000) coletaram oócitos 24 horas depois da administração do hCG e cultivados por 12-14 horas previamente à transferência ou transferidos com uma hora após a coleta. Com isso observaram que não houve diferença nas taxas de prenhez entre os oócitos cultivados in vitro $\mathrm{e}$ aqueles que foram imediatamente introduzidos no oviduto. Assim, concluíram que em equinos, os oócitos completam com sucesso o final do estágio de maturação dentro do oviduto.

Scott et al. (2001) ao realizarem um estudo com coleta de oócitos em éguas, observaram e compararam o potencial desenvolvimento de oócitos coletados de éguas in vivo e coletados de ovários obtidos em matadouro. Os oócitos coletados de folículos pré ovulatórios aspirados via transvaginal, guiado por ultrassom, resultaram em uma taxa de $82 \%$ de prenhez. Já oócitos que foram coletados de éguas durante o diestro e cultivados por 36-38 h resultaram em apenas 2 embriões de 29 oócitos coletados. Quando os oócitos foram coletados de éguas durante o diestro e diretamente introduzidos no oviduto da receptora, não resultaram em prenhez, sugerindo que pode ser que o final da maturação dos oócitos ocorrera no oviduto da receptoras, 
mas não a maturação completa do oócito. Os mesmos autores ao coletarem oócitos de ovários em matadouros, e cultivados in vitro por $36-38 \mathrm{~h}$ e transferidos para o oviduto resultaram em quatro prenhez de 40 oócitos (10\%).

Oócitos coletados de folículos pré ovulatório de éguas jovens e introduzidos em ovidutos também de éguas jovens resultam em alto desenvolvimento embrionário. Em oócitos cultivados in vitro, as taxas de prenhez são consideravelmente baixas, indicando que o sistema de maturação não é adequado para a maturação nuclear e citoplasmática (Scott et al., 2001).

Carnevale et al. (2001) avaliaram o resultado de três anos de um programa comercial utilizando as éguas mais velhas como doadora e observaram que os principais fatores que afetam nas taxas de transferência de oócito é a qualidade do oócito, idade da égua, e qualidade do sêmen. A taxa de prenhez foi de aproximadamente $30-40 \%$ por oócito transferido nesse programa comercial de TO.

\section{Recuperação de oócito in vivo}

O primeiro relato de coleta e transferência de oócitos in vivo pelo método de aspiração transvaginal guiada por ultra-som em animais domésticos foi em bovinos por Pieterse et al. (1988). Brück et al. (1992) foram os pioneiros na descrição da técnica em equinos. Esses autores relataram que se pode realizar a técnica de coleta de oócitos em éguas pela técnica de lavagem e aspiração folicular por via transvaginal, guiada por ultrassonografia. Os pesquisadores realizaram a punção de quatro folículos pré ovulatório e apesar de apenas um oócito ter sido recuperado, afirmaram que o método empregado mostrou-se rápido, preciso e atraumático.

Segundo Bézard (1997) a aspiração folicular em éguas por via transvaginal é considerada como um método não invasivo e que possibilita a realização da técnica no mesmo animal em diferentes ciclos.

A técnica de aspiração folicular e recuperação de oócitos devem ser realizadas com a égua contida e em estação, devem-se retirar as fezes do reto da mesma, fazer uma devida higienização do períneo e sedar o animal. Através da palpação transrectal cada ovário deve ser manipulado e posicionado contra o fundo da parede do fundo vaginal para que a imagem ultrassonográfica dos folículos possa ser obtida, com a utilização de uma probe ultrassonográfica convexa, acoplada a um suporte juntamente com a agulha de aspiração (Carnevale et al., 2004); Rodrigues et al., 2006). Normalmente, uma agulha de lúmen de $14 \mathrm{G}$ (Hinrichs et al., 1990) ou 16 G (Mari et al., 2005) é utilizada para a aspiração, portanto de calibre duplo (Rodrigues, 2006) para que aumente a taxa de recuperação de oócitos devido ao fato de se lavar o folículo e puncionar com solução de recuperação, fazendo com que o oócito se desprenda da parede do folículo com mais facilidade.

Após os ovários serem devidamente posicionados, pode-se observar a imagem do folículo a ser aspirado, em seguida a agulha é impulsionada suavemente contra fundo da vagina para que atravesse e alcance o folículo. Em seguida, o folículo é lavado e para evitar coagulação do material aspirado utiliza-se 100 $\mathrm{mL}$ de solução tamponada e $10 \mathrm{UI} / \mathrm{mL}$ de heparina a $37^{\circ} \mathrm{C}$ (McKinnon et al., 1997); ao aspirar o folículo o fluído folicular é armazenado no copo coletor.Utiliza-se níveis de pressão variando de $90 \mathrm{mmHg}$ a $400 \mathrm{mmHg}$; no entanto, a utilização de níveis muito altos pode causar lesões e desnudamento nos oócitos (Rodrigues, 2006). Uma das vantagens da aspiração folicular transvaginal é por ser um procedimento não cirúrgico e podem ser realizadas repetidas vezes, sem afetar a fertilidade das éguas submetidas ao procedimento (Silva et al., 2004). A técnica é considerada eficiente na obtenção de oócitos de éguas sub férteis, com patologias adquiridas, que apresentem históricos de problemas de infertilidade quando submetidas às biotecnologias de IA e TE (Fernandes et al., 2006).

Após ser realizada a aspiração, o fluido folicular é colocado em placa de Peri e realiza-se a busca dos oócitos e a classificação em estéreomicroscópio binocular. Localizados os gametas, os mesmos são transferidos para meios de cultura específicos, para receberem condições para serem transferidos imediatamente ou maturados in vitro, assim como também podem ser criopreservados (Carnevale et al., 2001, Preis et al., 2004).

Pode-se estimar o potencial de maturação, fecundação e capacidade de desenvolvimento do embrião de equinos por avaliação das características do complexo cumulus oophorus. Em geral, oócitos com maior viabilidade, possuem ooplasma homogêneo, com uma 
coloração marrom, granulações finas e apresentam-se completamente envolvidos por ao menos uma camada completa de células do cumulus (Curcio, 2005). Os resultados de taxas de recuperação encontrada por diferentes autores são diversos, assim como os equipamentos de ultra-som, os níveis de pressão utilizados na técnica e os modelos de agulhas utilizados nos procedimentos (Meintjes et al., 1994, Brück et al., 1997, Hinrichs et al., 1998).

Pycock (1996) reporta em seus estudos que a comparação entre as taxas de recuperação de oócitos em diferentes estudos torna-se impossível devido justamente, a diversidade de protocolos utilizados.

Existem vários fatores que influenciam na recuperação de oócitos, como terapias hormonais com gonadotróficas, fase do ciclo estral que a fêmea se encontra, frequência de realização de técnicas, tamanho do folículo e experiência do profissional que irá conduzir o procedimento (Fernandes et al., 2006). Segundo Deleuze et al. (2009), oócitos provenientes de folículos em desenvolvimento resultam em baixos índices de recuperação. Em equinos esse fato acontece porque no folículo existe uma camada de células da teca localizada abaixo da junção com o cumulus proporcionando uma maior aderência do oócito à parede folicular (Carnevale et al., 2004). No entanto, segundo Fernandes et al. (2006), à medida que o folículo vai se desenvolvendo, o índice de recuperação folicular torna-se maior devido ao afrouxamento das junções entre células do cumulus e da parede folicular. Essa maior facilidade de recuperação de oócitos nessa fase ocorre em decorrência do aumento das concentrações de LH em folículos pré ovulatórios assim como pela aplicação exógena de hormônios. Além da facilidade da recuperação de oócitos provenientes de folículos pré ovulatórios, os mesmos podem ser utilizados imediatamente sem a necessidade do cultivo e maturação in vitro (Carnevale et al., 2004).

Deleuze et al. (2009) após realizar 31 punções foliculares de éguas, foram obtidos um total de 158 oócitos de 284 folículos imaturos, resultando em uma taxa de recuperação de 55,6\%. Dos 158 oócitos, 47 foram utilizados imediatamente, 109 oócitos foram maturados in vitro, 40 oócitos foram usados para transferência intra-oviduto (TO) com uma taxa de embriões recuperados de $32,5 \%$. Jacobson et al. (2010) ao realizarem aspiração folicular em éguas, observou que houve efeito da égua no número de folículos puncionados, mas não na taxa de recuperação. $\mathrm{E}$ a administração de progesterona não teve efeito no número de folículos aspirados ou na taxa de recuperação de oócitos. Os resultados encontrados para número de folículos puncionados foi de $9,1 \pm 0,9(296 / 33)$ por éguas no grupo controle e 9,0 $\pm 0,7(300 / 33)$ por égua no grupo de administração de progesterona $(\mathrm{P})$. A quantidade de oócitos recuperados por égua por aspiração foi de 5,0 $\pm 0,6(166 / 33)$ por égua no grupo controle e 4,8 $\pm 0,5(157 / 33)$ por égua no grupo de P.

Segundo Galli et al. (2007) se cada ovário contem aproximadamente 5,3 folículos com diâmetro por volta de $5 \mathrm{~mm}$, e esses folículos forem puncionados, a taxa de recuperação é de 3,8 oócitos, correspondendo a $70 \%$ de eficiência na coleta.

\section{Maturação de oócitos in vitro}

Os primeiros dados de uma maturação in vitro realizada com sucesso em equinos foi relatada por Fulka \& Okolski (1981) quando coletaram oócitos de ovários de abatedouro e maturaram in vitro e posteriormente realizaram a transferência para o oviduto de éguas inseminadas.

Há uma limitação na realização da técnica em equinos devido à falta de abatedouros de equinos disponíveis, que forneça ovários de éguas para realização dos estudos, limitando o interesse em criadores e pesquisadores. A recuperação de oócitos de ovários de éguas apresenta como problemas técnicos a necessidade de excessiva lavagem do folículo para que haja desprendimento do complexo do cumulus do oócito. Essas dificuldades são refletidas no aumento do período de tempo e profissionais requeridos para coletar oócitos necessários para realização de um experimento (Galli et al., 2007). A necessidade de utilizar uma pressão maior de fluído no folículo para recuperar o oócito, é devido à justa conexão entre o cumulus e a membrana granulosa da parede do folículo (Hawley et al., 1995). Segundo o mesmo autor, em éguas, normalmente recupera-se oócitos cercados apenas pelas células da coroa, ou até mesmo oócitos recobertos com partes contendo células da granulosa, nesse caso indicando a realização de intensas lavagens seguida pela raspagem do folículo. Todas essas limitações representam a maior limitação para a pesquisa básica em oócitos equinos e para todas as 
tecnologias de fertilização in vitro e para inseminação intracitoplasmática (ICSI) (Galli et al., 2007).

Outro interessante aspecto peculiar dos oócitos de equinos é a frequente coleta de oócitos com cumulus expandido, quase um terço de oócitos recuperados. Em outras espécies como em ruminantes e suínos, quando são coletados oócitos com cumulus expandido, são descartados devido à sua baixa taxa de capacidade de desenvolvimento. No entanto, em equinos, oócitos com cumulus expandido maturam normalmente e tem normal desenvolvimento (Galli et al., 2007).

Galli et al. (2007) após coletarem oócitos, os mesmos foram selecionados de acordo com a morfologia do cumulus e transferidos para um meio de maturação por $24 \mathrm{~h}$, observaram que oócitos com cumulus expandido apresentaram mesma capacidade de completar a maturação e desenvolvimento ao estágio de blastocisto comparados com oócitos com cumulus compacto. Ao calcularem a taxa de maturação, foi observado também grande número de oócitos degenerados identificados após a maturação in vivo que é outra peculiaridade da espécie equina quando oócitos são coletados de ovários de abatedouro. Devido à falha em se obter resultados consistentes com a fertilização in vitro (FIV), muitas pesquisas estão se voltando para utilização de maturação de oócitos in vivo. Infelizmente o mesmo sucesso dos resultados reportados de FIV em outras espécies nunca ocorreu com a espécie equina, sendo poucos dados reportados nessa espécie (Palmer et al., 1990). Com isso há uma maior necessidade em realizar mais estudos com maturação de oócitos in vitro para otimizar a técnica.

Uma variedade de condições de maturação de oócitos é testada e avaliada usando diferentes meios de cultura como o TCM199 (Galli, 2002, Lagutina et al., 2005), B2 (Willis et al., 1991) e Ham's F10 (Shabpareh et al., 1993), G1 (Choi et al., 2002), DMEM-F12 e CZB (Choi et al., 2004) suplementados com diferentes concentrações de soro, hormônios ou fluido folicular. Essas condições tem resultado em taxas de maturação variando de 20 a $85 \%$. Um sistema de cultivo in vitro tem sido desenvolvido utilizando DMEM/F12, promovendo taxas de desenvolvimento de blastocistos similares aos observados in vivo (27 -38\%) (Hinrichs et al., 2005, Choi et al., 2006). Tremoleda et al. (2003) ao avaliarem e compararem o número de células entre embriões produzidos in vivo e in vitro ambos no dia 7 de desenvolvimento, observaram que embriões produzidos in vitro obtiveram menos número de células.

\section{Injeção de sêmen intracitoplasmática (ICSI)}

A aplicação da ICSI em equinos vem ultrapassando a barreira da ineficiência da FIV, resultando melhores resultados de prenhez utilizando oócitos maturados in vitro (Squires et al., 1996). A vantagem da ICSI comparada com a FIV é que a ICSI tem a habilidade de aumentar a oportunidade de utilização de garanhões que tenham sêmen com baixa motilidade e fertilidade in vivo. Lazzari et al. (2002) estudaram a capacidade de desenvolvimento de oócitos maturados in vitro e fertilizados por ICSI com sêmen congelado-descongelado de garanhões com sêmen de diferentes motilidade e fertilidade. Os mesmos autores observaram que não houve diferença na clivagem ou índice de desenvolvimento embrionário entre oócitos injetados com espermatozóides de garanhões com boa qualidade seminal ou com qualidade seminal ruim.

Galli et al. (2007) produziram quatro embriões de grau 1 por ICSI utilizando sêmen de baixa fertilidade e submetidos ao congelamento e descongelamento. Os embriões foram transferidos não cirurgicamente, dois embriões por receptora, os quais originaram prenhez dupla. Os resultados obtidos por Galli et al. (2007) indicam que a ICSI permite o uso de sêmen com baixa motilidade e fertilidade in vivo, desde que uma célula móvel seja selecionada para a injeção citoplasmática. Choi et al. (2006) realizaram inseminação intracitoplasmática com espermatozóides imóveis de sêmen submetidos a dois ciclos de congelamento e descongelamento, resultando em blastocistos.

\section{Considerações finais}

Apesar das dificuldades encontradas nas realizações das biotecnologias reprodutivas mais avançadas em equinos, a mesmas podem trazer bons resultados e perspectivas de uso, porém novas pesquisas são necessárias para que possam ser aprimoradas.

\section{Agradecimentos}

A CAPES pela concessão da bolsa de estudos. 


\section{Referências Bibliográficas}

Bézard, J. (1997). Aspects of in vivo fertilization of the equine oocyte. Arquivos da Faculdade de Veterinária, 25, 36-61.

Brück, I., Raun, K., Synnestedt, B. \& Greve, T. (1992). Follicle aspiration in the mare using a transvaginal ultrasound-guided technique. Equine Veterinary Journal, 24, 58-59.

Brück, I., Synnestvedt, B. \& Greve, T. (1997). Repeated trasnvaginal oocyte aspiration in unistimulated and FSH - treated mares. Theriogenology, 47, 1157-1167.

Carnevale, E., Maclellan, L., Silva, M., Checura, C., Scoggin, C. \& Squires, E. (2001). Equine sperm-oocyte interaction: results after intraoviductal and intrauterine inseminations of recipients for oocyte transfer. Animal Reproduction Science, 68, 305-314.

Carnevale, E., Maclellan, L., Silva, M., Scott, T. $\&$ Squires, E. (2000). Comparison of culture and insemination techniques for equine oocyte transfer. Theriogenology, 54, 981-987.

Carnevale, E., Silva, M., Panzani, D., Stokes, J. $\&$ Squires, E. (2005). Factors affecting the success of oocyte transfer in a clinical program for subfertile mares. Theriogenology, 64, 519-527.

Carnevale, E. M., Frank-Guest, B. L. \& Stokes, J. E. (2010). Effect of equine oocyte donor age on success of oocyte transfer and intracytoplasmic sperm injection. Animal Reproduction Science, 121, 258-259.

Carnevale, E. M. \& Ginther, O. J. (1995). Defective oocytes as a cause of subfertility in old mares. Biology and Reproduction Monograph, 1, 209-214.

Carnevale, E. M., Silva, M. A. C., Maclellan, L. J., Seidel, G. E. \& Squires, E. L. (2004). Use of parentage testing to determine optimum insemination time and culture media for oocyte transfer in mares. Reproduction, 128, 623-628.

Choi, Y., Love, C., Love, L., Varner, D., Brinsko, S. \& Hinrichs, K. (2002). Developmental competence in vivo and in vitro of in vitro-matured equine oocytes fertilized by intracytoplasmic sperm injection with fresh or frozen-thawed spermatozoa. Reproduction, 123, 455-465.
Choi, Y., Love, C., Varner, D. \& Hinrichs, K. (2006). Equine blastocyst development after intracytoplasmic injection of sperm subjected to two freeze-thaw cycles. Theriogenology, 65, 808-819.

Choi, Y., Love, L., Varner, D. \& Hinrichs, K. (2004). Factors affecting developmental competence of equine oocytes after intracytoplasmic sperm injection. Reproduction, 127, 187-194.

Confederação da Agricultura e Pecuária do Brasil. (2004). Estudo do complexo do agronegóciocavalo. Brasília: CNA, Coletânea Estudos Gleba, n.39.

Curcio, B.R. (2005). Maturação e vitrificação de ovócitos equinos incubados em meios contendohormônio do crescimento e fator de crescimento semelhantes à insulina-I. 75f. Dissertação (Mestrado em Ciências). Pelotas: Faculdade de Medicina Veterinária, Universidade Federal de Pelotas.

Deleuze, S., Goudet, G., Caillaud, M., Lahuec, C. \& Duchamp, G. (2009). Efficiency of embryonic development after intrafollicular and intraoviductal transfer of in vitro and in vivo matured horse oocytes. Theriogenology, 72, 203-209.

Escola Superior de Agricultura Luiz de Queiroz ESALQ. (2006). Estudo do Complexo Agronegócio Cavalo no Brasil. Brasília: CEPEA,. 68p, Coletânea de Estudos Gleba; n. 40.

Fernandes, C., Peres, K., Alvarenga, M. \& Landim-Alvarenga, F. (2006). The use of transmission electron microscopy and oocyte transfer to evaluate in vitro maturation of equine oocytes in different culture conditions. Journal of Equine Veterinary Science, 26, 159-167.

Fulka, J. \& Okolski, A. (1981). Culture of horse oocytes in vitro. Journal of Reproduction and Fertility, 61, 213-215.

Galli, C. (2002). Frozen-thawed embryos produced by Ovum Pick Up of immature oocytes and ICSI are capable to establish pregnancies in the horse. Theriogenology, 58, 705-708.

Galli, C., Colleoni, S., Duchi, R., Lagutina, I. \& Lazzari, G. (2007). Developmental competence of equine oocytes and embryos obtained by in vitro procedures ranging from 
in vitro maturation and ICSI to embryo culture, cryopreservation and somatic cell nuclear transfer. Animal Reproduction Science, 98, 39-55.

Hawley, L. R., Enders, A. C. \& Hinrichs, K. (1995). Comparison of equine and bovine oocyte-cumulus morphology within the ovarian follicle. Biology of Reproduction Monograph, 1, 243-252.

Hinrichs, K., Betschart, R., McCue, P. \& Squires, E. (1999). Effect of timing of follicle aspiration on pregnancy rate after oocyte transfer in mares. Journal of reproduction and fertility, 56, 493-498.

Hinrichs, K., Choi, Y., Love, L., Varner, D., Love, C. \& Walckenaer, B. (2005). Chromatin configuration within the germinal vesicle of horse oocytes: changes post mortem and relationship to meiotic and developmental competence. Biology of Reproduction, 72, 1142-1150.

Hinrichs, K., Kenney, D. \& Kenney, R. (1990). Aspiration of oocytes from mature and immature preovulatory follicles in the mare. Theriogenology, 34, 107-112.

Hinrichs, K., Matthews, G., Freeman, D. \& Torello, E. (1998). Oocyte transfer in mares. Journal of the American Veterinary Medicine Association, 212, 982-986.

Imboden, I., Janett, F., Burger, D., Crowe, M., Hässig, M. \& Thun, R. (2006). Influence of immunization against $\mathrm{GnRH}$ on reproductive cyclicity and estrous behavior in the mare. Theriogenology, 66, 1866-1875.

Jacobson, C. C., Choi, Y.-H., Hayden, S. S. \& Hinrichs, K. (2010). Recovery of mare oocytes on a fixed biweekly schedule, and resulting blastocyst formation after intracytoplasmic sperm injection. Theriogenology, 73, 1116-1126.

Lagutina, I., Lazzari, G., Duchi, R., Colleoni, S., Ponderato, N., Turini, P., Crotti, G. \& Galli, C. (2005). Somatic cell nuclear transfer in horses: effect of oocyte morphology, embryo reconstruction method and donor cell type. Reproduction, 130, 559-567.

Lazzari, G., Crotti, G., Turini, P., Duchi, R., Mari, G., Zavaglia, G., Barbacini, S. \& Galli, C. (2002). Equine embryos at the compacted morula and blastocyst stage can be obtained by intracytoplasmic sperm injection (ICSI) of in vitro matured oocytes with frozen-thawed spermatozoa from semen of different fertilities. Theriogenology, 58, 709-712.

Mari, G., Barbara, M., Eleonora, I. \& Stefano, B. (2005). Fertility in the mare after repeated transvaginal ultrasound-guided aspirations. Animal Reproduction Science, 88, 299-308.

McKinnon, A., Carnevale, E., Squires, E., Voss, J. \& Seidel, G. (1988). Heterogenous and xenogenous fertilization of in vivo matured equine oocytes. Journal of Equine Veterinary Science, 8, 143-147.

McKinnon, A., Perriam, W., Lescun, T., Walker, J., VASEY, J. \& Trigg, T. (1997). Effect of a GnRH analogue (Ovuplant), hCG and dexamethasone on time to ovulation in cycling mares. World Equine Veterinary Review, 2, 3.

Meintjes, M., Bellow, M., Broussard, J., Paccamonti, D., Eilts, B. \& Godke, R. (1994). Repeated transvaginal ultrasound-guided oocyte retrieval from pregnant mares. Theriogenology, 41, 255.

Palmer, E., Bezard, J., Magistrini, M. \& Duchamp, G. (1990). In vitro fertilization in the horse. A retrospective study. Journal of Reproduction and Fertility, 44, 375-384.

Pieterse, M., Kappen, K., Kruip, T. A. \& Taverne, M. (1988). Aspiration of bovine oocytes during transvaginal ultrasound scanning of the ovaries. Theriogenology, 30, 751-762.

Preis, K. A., Carnevale, E., Silva, M. C., Brienza, V. C., Gomes, G., Maclellan, L. \& Squires, E. (2004). In vitro maturation and transfer of equine oocytes after transport of ovaries at 12 or 22 C. Theriogenology, 61, 1215-1223.

Pycock, J. F. (1996). Recovery of oocytes using transvaginal ultrasound in the mare: current equipment, techniques and applications. Arquivo da Faculdade de Veterinária, 24, 148-167.

Ray, B., Squires, E., Cook, N., Tarr, S., Jasko, D. \& Hossner, K. (1994). Pregnancy following gamete intrafallopian transfer in the mare. Journal of Equine Veterinary Science, 14, 27 30.

Ribeiro, B., Love, L., Choi, Y. \& Hinrichs, K. (2008). Transport of equine ovaries for 
assisted reproduction. Animal Reproduction Science, 108, 171-179.

Rodrigues, R. (2006). Aspiração folicular por via transvaginal guiada por ultra-som em equinos. Dissertação (Mestrado em Ciência Veterinária). Porto Alegre: Faculdade de Medicina Veterinária, Universidade Federal do Rio Grande do Sul.

Scott, T. J., Carnevale, E., Maclellan, L., Scoggin, C. \& Squires, E. (2001). Embryo development rates after transfer of oocytes matured in vivo, in vitro, or within oviducts of mares. Theriogenology, 55, 705-715.

Shabpareh, V., Squires, E., Seidel, G. \& Jasko, D. (1993). Methods for collecting and maturing equine oocytes in vitro. Theriogenology, 40, 1161-1175.

Silva, M., Carnevale, E., Maclellan, L., Preis, K., Seidel, G. \& Squires, E. (2004). Oocyte transfer in mares with intrauterine or intraoviductal insemination using fresh, cooled, and frozen stallion semen. Theriogenology, 61, 705-713.

Squires, E., Carnevale, E., McCue, P. \& Bruemmer, J. (2003). Embryo technologies in the horse. Theriogenology, 59, 151-170.

Squires, E.L., Seidel, J.R. (1995). Collection and transfer of equine embryos.Animal
Reproduction Biotechnology Laboratory. Colorado State University, Fort Collins, CO.

Squires, E., Wilson, J., Kato, H. \& Blaszczyk, A. (1996). A pregnancy after intracytoplasmic sperm injection into equine oocytes matured in vitro. Theriogenology, 1, 306.

Tremoleda, J. L., Stout, T. A., Lagutina, I., Lazzari, G., Bevers, M. M., Colenbrander, B. \& Galli, C. (2003). Effects of in vitro production on horse embryo morphology, cytoskeletal characteristics, and blastocyst capsule formation. Biology of Reproduction, 69, 1895-1906.

Willis, P., Caudle, A. \& Fayrer-Hosken, A. (1991). Equine oocyte in vitro maturation: influences of sera, time, and hormones. Molecular reproduction and development, 30, 360-368.

Recebido em Outubro 18, 2015

Aceito em Dezembro 2, 2015

License information: This is an open-access article distributed under the terms of the Creative Commons Attribution License, which permits unrestricted use, distribution, and reproduction in any medium, provided the original work is properly cited 